\title{
Echocardiographic detection of a metastatic right atrial mass in a patient with previously un-diagnosed hepatocellular carcinoma who presents with dyspnea
}

\author{
Ber-Ren Fang ${ }^{1,4^{*}}$, Chin-Yew Lin $^{2,4}$, I-Ping Yen ${ }^{3,4}$ \\ ${ }^{1}$ Division of Cardiology, Cardinal Tien Hospital Yung Ho Branch, Yung Ho City, Taipei County, Taiwan, China; \\ *Corresponding Author: kaec15@MS27.hinet.net; \\ ${ }^{2}$ Department of Pathology, Cardinal Tien Hospital, Yuan-Ho City, Taipei County, Taiwan, China; \\ ${ }^{3}$ Division of Cardiology, Cardinal Tien Hospital, Xian Dian City, Taipei County, Taiwan, China; \\ ${ }^{4}$ Fu-Jen Catholic University, College of Medicine, Xin Zhung City,Taipei County, Taiwan, China.
}

Received 3 January 2011; revised 19 January 2011; accepted 29 January 2011

\begin{abstract}
A 69-year-old woman had experienced resistant edema of lower extremities and progressive dyspnea on exertion for two months. The patient visited our emergency room owing to exacerbation of her dyspnea symptom. Echocardiography demonstrated a mobile mass in the right atrium. Transesophageal echocardiography revealed a right atrial mass arising from the inferior vena cava which was partially mobile. The patient underwent urgent open heart surgery with resection of the right atrial mass and curettage of the tumor thrombus in the inferior vena cava. Histologic examination of the resected right atrial mass revealed the features of metastatic hepatocellular carcinoma. Subsequent work-up revealed that alpha-fetoprotein level was $3780 \mathrm{ng} / \mathrm{ml}$. Abdominal echocardiography showed a tumor mass in the right lobe of the liver. The post-operative course was complicated by pneumonia, sepsis, and multiorgan failure. The patient died 48 days after surgery.
\end{abstract}

Keywords: Hepatocellular Carcinoma; Right Atrial Metastasis; Echocardiography

\section{INTRODUCTION}

Intracardiac metastasis of hepatocellular carcinoma (HCC) is rare. Before the era of echocardiography, antemortem diagnosis of this condition was difficult and the condition was usually an incidental finding during autopsy. However, since the widespread application of two-dimensional and transesophageal echocardiography in general practice, diagnosis of this condition has increased [1-3]. This study presents a case of previously un-diagnosed HCC presenting with dyspnea and resistant leg edema. Echocardiography detected a right atrial mass that was removed surgically and was proven histologically to be a metastatic HCC.

\section{CASE REPORT}

A 69-year-old woman had experienced resistant edema of the lower extremities and progressive dyspnea on exertion for two months. The patient felt exacerbation of her dyspnea symptom lasting a few days, and was referred to our emergency-department by a local doctor. In the emergency room, physical examination revealed blood pressure 122/63 $\mathrm{mmHg}$, and pulse rate 84 beats per minute. The sclera was not icteric, conjunctiva was slightly pale, and the jugular veins were engorged. A grade 2/6 systolic murmur was heard at the left lower sternal border, and significant edema of the lower extremities was noted. Chest x-ray film revealed slight cardiomegaly. Moreover, electrocardiography demonstrated normal sinus rhythm. White blood cell count was $7700 / \mathrm{mm}^{3}$; hemoglobin was $10.1 \mathrm{gm} / \mathrm{dl}$; blood urea nitrogen was $24 \mathrm{mg} / \mathrm{dl}$, and creatinine was $1.8 \mathrm{mg} / \mathrm{dl}$. Echocardiography was arranged to investigate the possible cardiac cause of dyspnea and the nature of the cardiac murmur. Transthoracic echocardiography demonstrated a mass in the right atrium. The mass was partially mobile, and may prolapse into the right ventricle during the diastolic phase and moreover may return to the right atrium during the systolic phase. The mass appeared to arise from the junction of the right atrium and the inferior vena cava (Figure 1). Doppler echo demonstrated evidence of mild tricuspid stenosis and regurgitation. Since the presence of the right atrial mass was considered 

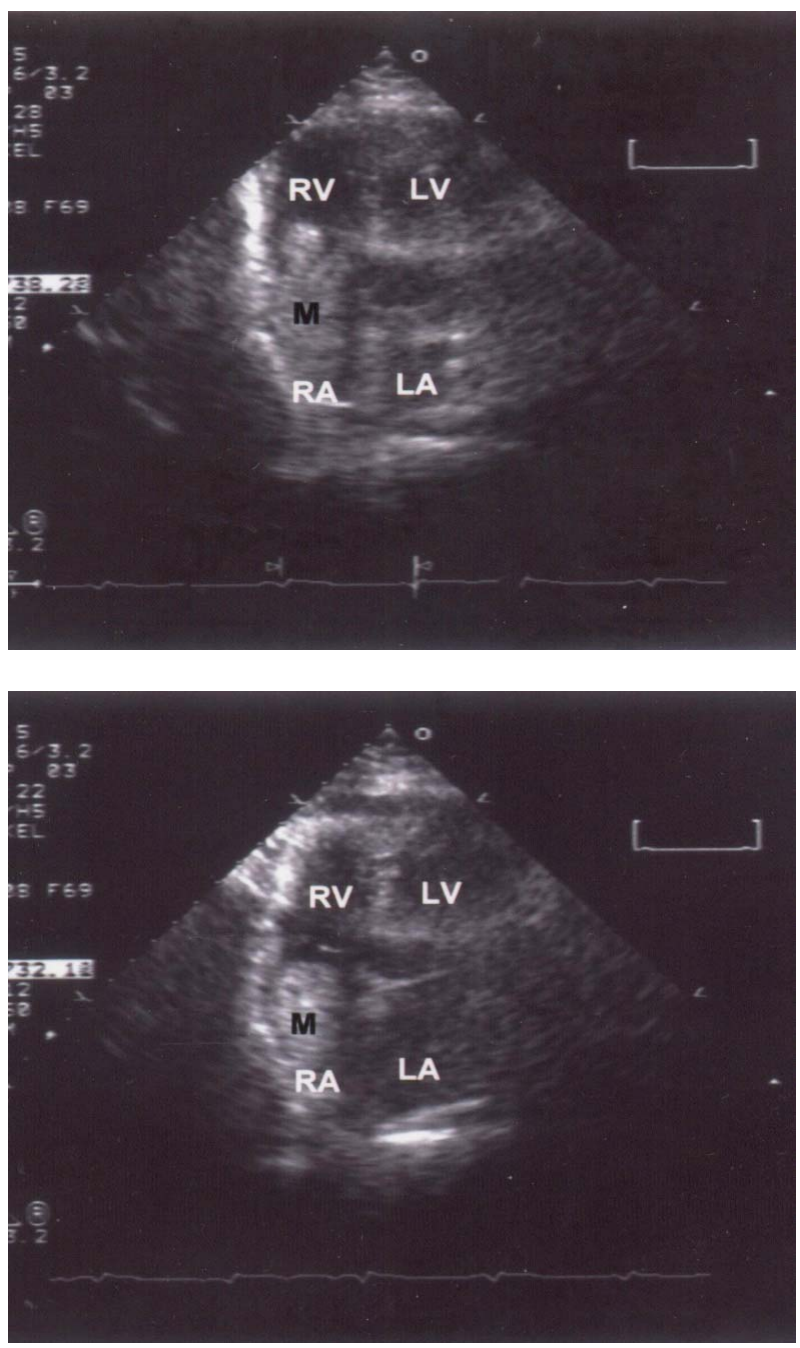

Figure 1. Apical four chamber view of two-dimensional echocardiography demonstrating a mobile mass in the right atrium. The mass may prolapse into the right ventricle during the diastolic phase (upper panel) and may return to the right atrium during systolic phase (lower panel). Abbreviations: LA, left atrium; LV, left ventricle; $M$, mass; RA, right atrium; RV, right ventricle.

a surgical emergency, the patient underwent urgent open heart surgery. Immediately prior to surgery, transesophageal echocardiography revealed a right atrial mass arising from the inferior vena cava which was partially mobile (Figure 2). On surgery, a mass measuring $8 \times 5 \times 3 \mathrm{~cm}$ and arising from the inferior vena cava was noted and excised. Curettage of the tumor thrombus in the inferior vena cava was performed as low as possible. Histologic examination of the excised right atrial mass revealed features of metastatic HCC (Figure 3). Workup for HCC was subsequently performed. ALT was 11 $\mathrm{u} / \mathrm{L}$ (normal $<36 \mathrm{u} / \mathrm{L}$ ), AST was $33 \mathrm{u} / \mathrm{L}$ (normal $<34 \mathrm{u} / \mathrm{L}$ ), albumin was $2.7 \mathrm{gm} / \mathrm{dl}$, HBS Ag was positive, anti-HC antibody was negative, and alpha-fetoprotein was 3789

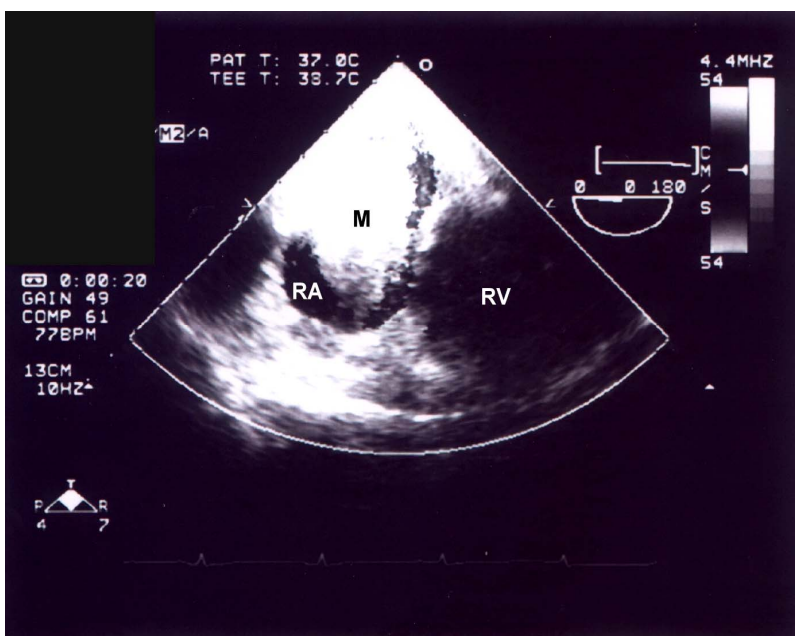

Figure 2. Transesophageal echocardiography displaying a mass in the right atrium. The mass arises from the junction of the right atrium and the inferior vena cava. Abbreviations: $M$, mass; RA, right atrium; RV, right ventricle.

$\mathrm{ng} / \mathrm{ml}$ (normal <20 ng/ml). Abdominal echocardiography identified a tumor mass measuring $8.0 \times 7.8 \mathrm{~cm}$ in the right lobe of the liver. Liver cirrhosis and ascites were also noted. The post-operative course was complicated by pneumonia, sepsis, respiratory failure and renal failure. The patient died 48 days after surgery.

\section{DISCUSSION}

The reported incidence of right atrial metastasis in hepatocellular carcinoma ranges from $0.67 \%$ to $4.1 \%$ $[4,5]$. According to autopsy findings, hepatocellular carcinoma tend to extend from the hepatic vein to the inferior vena cava and then into the right atrium. Kato et al. [4] described the autopsy findings in five patients with HCC who had tumor metastasis to the right atrium. In four of five patients in that series the tumor thrombus completely occluded the right hepatic vein and inferior vena cava and extended into the right atrium. Moreover, in another patient the hepatic vein was unaffected but the tumor thrombus was partially occluding the inferior vena cava and extending into the right atrium. In another autopsy series of 18 patients with HCC who had right atrial metastasis reported by Kojiro et al. [5], tumor thrombus involving the hepatic vein and the inferior vena cava was found in 15 (83.3\%) of the 18 cases. The presence of tumor thrombus in the inferior vena cava and right atrium may hinder venous return to the right heart, thus reducing cardiac output, and can also cause "secondary Budd-Chiari syndrome" which is characterized by diuretic resistant leg edema, ascites and dilatation of abdominal wall veins [6]. In the present case, the patient experienced progressive dyspnea on exertion and diuretic resistant leg edema. These symptoms and signs are 

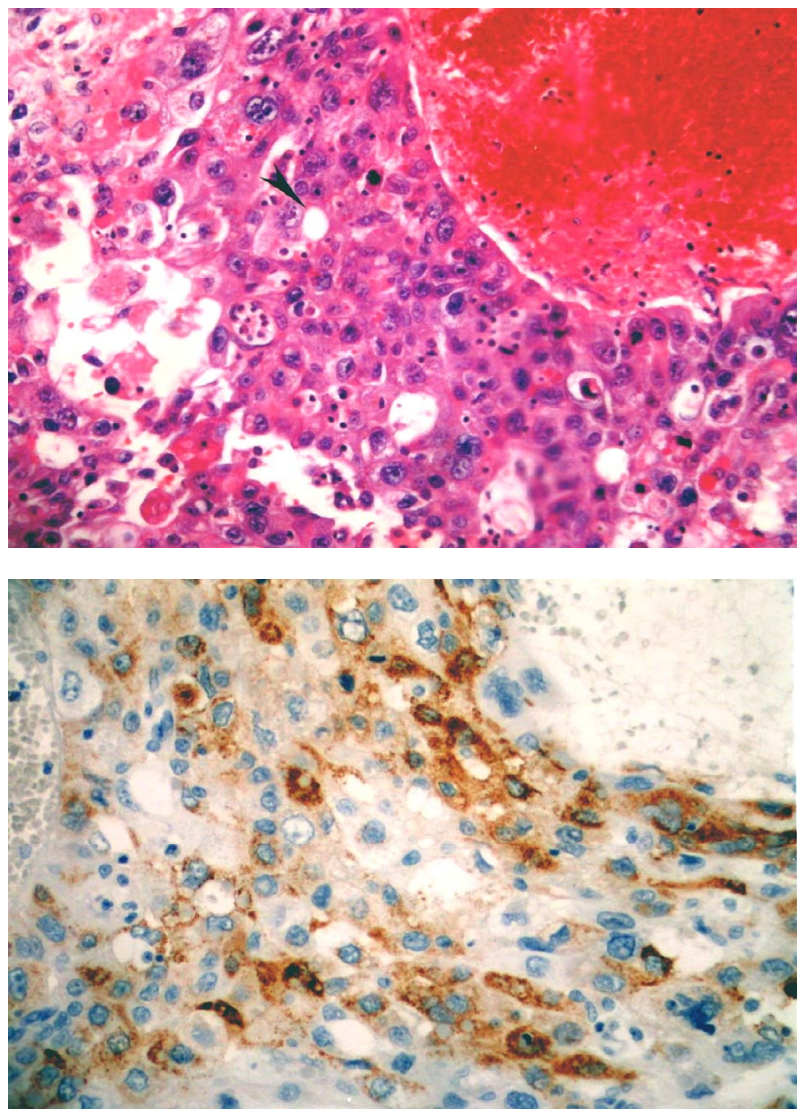

Figure 3. Histopathologic features of resected right atrial mass. Upper panel: This photograph demonstrating diffusely infiltrating neoplastic cells which appear in trabecular or sheet patterns of growth with occasional pseudoglandular formation (arrow-head). The malignant cells have distinct cellular borders and pleomorphic nuclei with prominent nucleoli (Hematoxylin-eosin stain, original magnification X 200). Lower panel: The immunohistochemical staining for anti-HepPar 1 showing strongly and diffusely positive in the tumor cells, confirming the diagnosis of metastatic hepatocellular carcinoma. (Immunoperoxidase staining with HepPar-1, original magnification X 200).

caused by reduced cardiac output and secondary BuddChiari syndrome. Echocardiography plays an important role in the detection of intracardiac mass. The characteristics and site of attachment of the mass may offer important information regarding the nature of the right atrial mass. Cardiac myxoma, which are rarely located in the right atrium, are generally highly mobile, and are mostly attached to the interatrial septum. Thrombus generally is fixed to the atrial wall, but also can be mobile. Meanwhile, metastatic tumor mass is generally fixed, but also can be mobile and is largely adherant to the junction of the right atrium and the inferior vena cava. Since tumor thrombus in the inferior vena cava is common in patients with HCC and right atrial metastasis, whenever an right atrial mass is detected by echocardi- ography, the inferior vena cava should be carefully scanned to exclude the possibility of cardiac involvement in malignancy [7]. However, transesophageal echocardiography gives more information about precise site of attachment to the atrial wall or interatrial septum and clearly illustrates the relationship with the inferior and superior vena cava [8]. When the right atrial mass is mobile as in the present case, the mass may move back and forth through the tricuspid orifice, causing systolic and/or diastolic murmur. The presence of thrombi (either attached or free-floating) in the right cardiac chambers carries the risk of pulmonary embolism and sudden death [9]. In most of these cases, pulmonary embolism has been observed to occur within a few days after echocardiographic diagnosis [9]. Ehrich et al. [10] reported a patient with HCC and right atrial metastasis who suffered a sudden drop in blood pressure. Surgical excision of the metastatic right atrial mass caused symptomatic relief, and the patient was still alive one year after surgery. Masaki et al. [11] described two patients with HCC and right atrial metastasis. Surgical removal of the tumor thrombus improved the dyspnea symptoms in each case. The subsequent course revealed one patient that survived for eight months and was able to return to work, while the other patient had unsatisfactory recovery and died suddenly one month after surgery. In the present case the patient died 48 days after surgery owing to sepsis and multi-organ failure. The rationale for surgical treatment in these patients is as follows: first, prevention of sudden death possibly due to massive pulmonary embolism [1,9,12], second, improvement of life quality by ameliorating the symptoms of reduced cardiac output (dyspnea) and secondary Budd-Chiari syndrome [10-12].

In conclusion, diagnosing right atrial metastasis in HCC is difficult because the symptoms and signs are nonspecific. However, right atrial metastasis should be considered when patients with HCC present with dyspnea, abnormal cardiac sounds and Budd-Chiari syndrome. Echocardiography is the most useful diagnostic tool for detecting metastatic right atrial mass.

\section{REFERENCES}

[1] Chua, S.O., Chiang, C.W., Lee, YS, Liaw, Y.F., Chang, C.H. and Hung, J.S., (1989) Echocardiographic findings of mobile atrial hepatocellular carcinoma report of five cases. Journal of Ultrasound in Medicine, 8, 347-352.

[2] Camp, G.V., Abdulsater, J., Cosyns, B., Liebens, I. and Vamdenbossche, J.L., (1994) Transesophageal echocardiography of right atrial metastasis of a hepatocellular carcinoma. Chest, 105, 945-947. doi:10.1378/chest.105.3.945

[3] Vlasseros, I., Tapanlis, E., Katsaros, A., Katsaros, A., Kountouras, D. and Gialafos, I. (2003) Metastatic hepa- 
tocellular carcinoma into the right atrium and ventricle: echocardiographic diagnosis and follow-up. Echocardiography, 20, 387-388. doi:10.1046/j.1540-8175.2003.03047.X

[4] Kato, Y., Tanaka, N., Kobayashi, K., Ikeda, T., Hattori, N. and Nonomura, A. (1983) Growth of hepatocellular carcinoma into the right atrium: report of five cases. Annals of Internal Medicine, 99, 472-474.

[5] Kojiro, M., Nakahara, H., Sugihara, S., Murakami, T., Nakashima, T. and Kawasaki, H. (1984) Hepatocellular carcinoma with intra-atrial tumor growth. Archives of Pathology \& Laboratory Medicine 108, 989-992.

[6] Takeuchi, J., Takada, A., Hasumura, Y., Matsuda, Y. and Ikegami, F. (1971) Budd-Chiari syndrome associated with obstruction of the inferior vena cava: a report of seven cases. American Journal of Medicine. 51, 11-20. doi:10.1016/0002-9343(71)90319-6

[7] Oh, J.K., Seward, J.B. and Tajik, A.J. (1999) The Echo Manual, 2th Edition, Lippincott Williams \& Wilkins, Philadelphia.

[8] Mugge, A., Daniel, W.G., Haverich, A. and Lichtlen, P.R., (1991) Diagnosis of noninfective mass lesions by two-dimensional echocardiography: comparison of the trasthoracic and transesophageal approaches. Circulation,
83, 70-78.

[9] Farfel, Z., Shechter, M., Vered, Z., Rath, S., Goor, D. and Gafni, J. (1987) Review of echocardiographically diagnosed right heart entrapment of pulmonary emboli-intransit with emphasis on management. American Heart Journal, 113, 171-178. doi:10.1016/0002-8703(87)90026-3

[10] Ehrich, D.A., Widmann, J.J., Berger, R.L. and Abelmann, W.H. (1975) Intracavitary cardiac extension of Hepatoma. The Annals of Thoracic Surgery, 19, 206-211. doi:10.1016/S0003-4975(10)64004-0

[11] Masaki, N., Hayashi, S., Maruyama, T., Okabe, H., Matsukawa, M., Unno, J., et al., (1994) Marked clinical improvement in patients with hepatocellular carcinoma by surgical removal of extended tumor mass in right atrium and pulmonary arteries. Cancer Chemotherapy and Pharmacology, 33, suppl 1, 7-11. doi:10.1007/BF00686660

[12] Fujisaki, M., Kurihara, E., Kikuchi, K., Nishikawa, K. and Uematsu, Y. (1991) Hepatocellular carcinoma with tumor thrombus extending into the right atrium: report of a successful resection with the use of cardiopulmonary bypass. Surgery, 109, 214-219. 